Journal of Advanced Research in Fluid Mechanics and Thermal Sciences

Journal homepage: www.akademiabaru.com/arfmts.html ISSN: 2289-7879

\title{
Ferroconvection in an Anisotropic Porous Medium with Variable Gravity
}

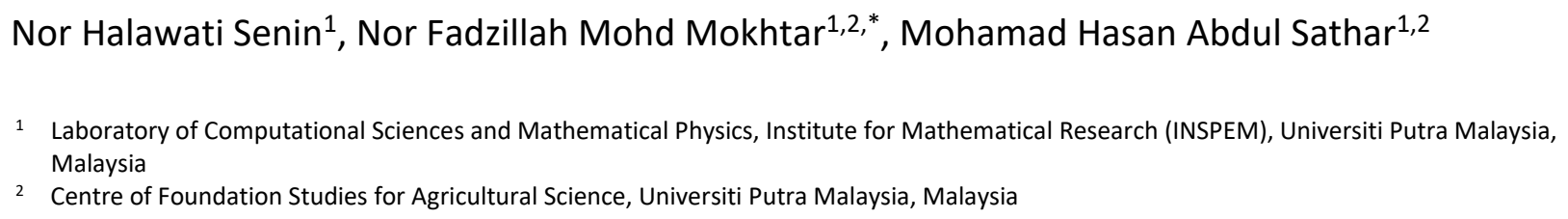

ARTICLE INFO

Received 26 December 2019

Received in revised form 31 January 2020

Accepted 24 March 2020

Available online 27 May 2020

\section{Article history:}

\section{ABSTRACT}

A linear stability assessment was performed to study the impact of internal heating and variable gravity in an anisotropic porous medium of a ferrofluid layer system on the onset of Benard-Marangoni convection. The system is heated from below with both the lower and upper limits are considered as completely insulated to the disturbance of the temperature. The eigenvalue problem is solved by using regular perturbation technique to obtain the critical Marangoni number and also the critical thermal Rayleigh number. It is noted that the increase of value anisotropic permeability, Darcy number and also magnetic number will enhance the convection of the system while the increasing values of anisotropic thermal diffusivity will help to stabilize the system.

Keywords:

Ferrofluid; Anisotropic; Variable Gravity
Copyright @ 2020 PENERBIT AKADEMIA BARU - All rights reserved

\section{Introduction}

Ferrofluid or also known as magnetic fluid is a non-electric carrier fluid that includes small particles of strong ferromagnetic materials [1]. Kaiser and Miskolczy [2] state that ferrofluid has a special feature which is it can maintain it fluid properties in the existence of magnetic field and the magnetic properties of ferrofluid can be affected by the composition, distribution and also volume concentration. Previously ferrofluid is known in the rocket fuel by NASA and currently ferrofluid been used in a various field such as in electric devices, mechanical engineering, medical applications and optic. There have been tremendous studies on the convection of ferrofluid. Stiles and Kagan [3] examined the instability of ferroconvection in a strong magnetic field. The impact of the vertical magnetic field in ferrofluid was researched by Hennenberg et al., [4]. Mokhtar and Arifin [5] had the impacts of feedback control in the ferrofluid layer system. Laroze et al., [6] employed chaos study in

\footnotetext{
* Corresponding author.

E-mail address: norfadzillah.mokhtar@gmail.com (Nor Fadzillah Mohd Mokhtar)
}

https://doi.org/10.37934/arfmts.71.2.5668 
ferrofluid. Laroze et al., [7] explored the instability of viscoelastic ferrofluid. Recently, the impact of magnetic field dependent viscosity in a ferrofluid was proved by Prakash et al., [8].

Marangoni convection can be understood as convection that considered surface tension in the study. The existence of surface tension cause the fluid flow from the area that has low surface tension. Nield [9] propose the idea of combination between surface tension forces and buoyancy forces study or usually name as Marangoni-Benard convection. The studied of Marangoni convection with a deformable surface had been done by McCaughan and Bedir [10]. In Marangoni-Benard convection, Hennenberg et al., [11] recorded a porous medium with Darcy law. Rudraiah and Prasad [12] had examined Brinkman's model in a porous medium. Saghir et al., [13] investigated dual-layer studies on the onset of Marangoni convection. Shivakumara et al., [14] employed BrinkmanForchheimer-Lapwood extended Darcy model on the onset of Marangoni convection. Feedback control effect with a deformable surface in a variable viscosity fluid had been studied by Arifin and Abidin [15]. Marangoni convection of porous medium with a Biot number had been studied by Zhao et al., [16]. Dual-layer fluid with the impact of internal heating in an upper boundary that is set to be deformable on the Marangoni-Benard convection had been demonstrated by Mokhtar et al., [17].

The study of convection that involving porous medium had been done widely, most of the porous medium studied previously are considering isotropic porous medium. Previously, Mahad et al., [18] applied an isotropic model with physical invariant for a heart valve leaflet because the material has only one direction toward the fiber direction. Since the formation of anisotropic porous medium can be happen naturally thus a lot of materials are considered as an anisotropic porous medium such as wood, carbonate rock and also composite. Degan and Vasseurt [19] demonstrated the convection of an anisotropic medium that oblique to gravity. Sekar et al., [20] investigated the convection of ferrofluid in an anisotropic porous medium. Marangoni-Benard convection problem in the anisotropic porous medium had been examined by Shivakumara et al., [21]. The study of anisotropic with modified Brinkman Darcy flow model had been employed by Nanjundappa et al., [22] in a ferrofluid system. Shivakumara et al., [23] study the impact of internal heating in an anisotropic medium in Marangoni-Benard convection. Bhadauria [24] also study the anisotropic porous medium with the effect of internal heating on the onset of double-diffusive convection. Capone et al., [25] studied an anisotropic and non-homogeneous porous medium in a linear and non-linear stability study. Soret-driven convection in an anisotropic porous ferrofluid layer system had been studied by Sekar et al., [26]. Recently Sun et al., [27] studied the impacts of an external magnetic field in a ferrofluid while Zarifah et al., [28] examined the temperature profile in a binary fluid with anisotropic porous medium.

The ideas of gravity are well known in the theoretical investigation, a lot of studies related to variable gravity had been done before to justify a convection phenomenon in a large scale such as in the ocean and mantle. Rionero and Straughan [29] investigated the combined effect of internal heating and variable gravity in convection of a porous medium. The effect variable gravity and internal heating with additionally inclined temperature gradient in a porous medium had been done by Alex et al., [30]. Chand [31] investigated rotating Maxwell of a visco-elastic porous medium with the additional effect of variable gravity. Bala and Chand [32] employed Brinkman porous medium with variable gravity of ferrofluid. Combination effect of rotating and variable gravity of porous nanofluid layer had been reported by Chand et al., [33]. Varshney [34] investigated on the stability on the convection of porous medium with the effect of gravity.

The aim of this paper is to investigate the onset of Marangoni-Benard convection with the impact of the variable gravity in an anisotropic porous medium. We assumed that the layer of ferrofluid is heated from below and that the conditions of the lower-upper boundary are considered to be a rigidfree boundary. Using a regular perturbation technique will solve the resulting eigenvalue problem. 


\section{Methodology}

We considered a horizontal ferrofluid layer system is heated from below as shown in Figure 1. The lower boundary is set to be rigid while the upper boundary is set to be free. Both of the boundaries are fixed to be constant but the temperature of the lower bound is higher compared to the upper bound. The ferrofluid layer scheme is applied by gravitational force $h(0,0,-h(z))$ where $h(z)=(1+\lambda) h$ and $\lambda$ are the parameter of variable gravity.

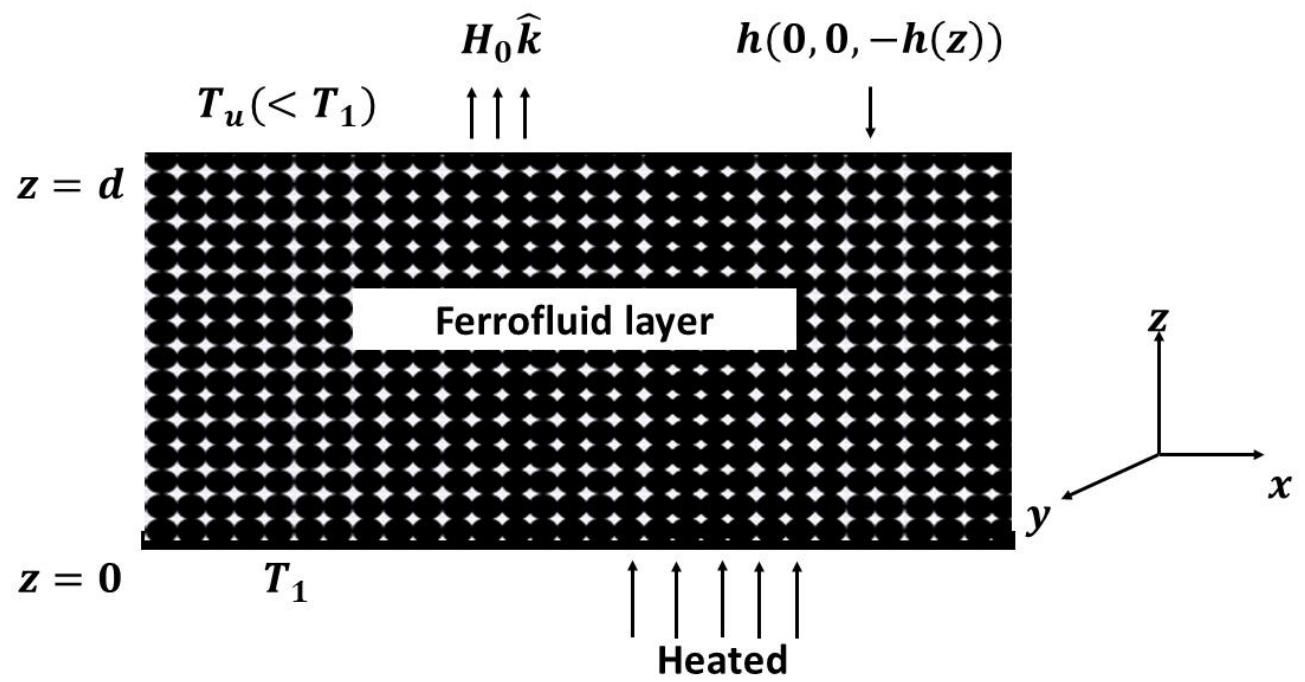

Fig. 1. Model of an anisotropic porous medium in ferrofluid layer system

The surface tension, $\sigma$ and density of the fluid, $\rho$ are in the form of

$\sigma=\sigma_{0}-\sigma_{T}\left(T-T_{0}\right)$

$\rho=\rho_{0}\left[1-\alpha_{t}\left(T-T_{0}\right)\right]$,

where $\sigma_{0}, \rho_{0}$ and $T_{0}$ are reference value of surface tension, density and temperature respectively while $\sigma_{T}$ is the rate of change of the surface tension at the temperature $T$. The surface tension and density are assumed vary linearly with the temperature. By referring to Nanjundappa et al., [22], the governing equations are as follows

$\nabla \cdot \vec{q}=0$

$\frac{\rho_{0}}{\varepsilon}\left[\frac{\partial \vec{q}}{\partial t}+(\vec{q} \cdot \nabla) \vec{q}\right]=-\nabla p+\rho h+\mu_{0}(\vec{M} \cdot \nabla) \vec{H}+\mu k^{-1} \vec{q}$

$\varepsilon\left[\rho_{0} C_{V, H}-\mu_{0} \vec{H} \cdot\left(\frac{\partial \vec{M}}{\partial T}\right)_{V, H}\right] \times \frac{D T}{D t}+(1-\varepsilon)\left(\rho_{0} C\right) \frac{\partial T}{\partial t}+\mu_{0} T\left(\frac{\partial \vec{M}}{\partial T}\right)_{V, H} \cdot \frac{D \vec{H}}{D t}=k_{1} \nabla^{2} T$

Here $\vec{q}=(u, v, w)$ is the velocity vector, $\mu$ is the dynamic viscosity, $\mu_{0}$ is the magnetic permeability of vacuum, $p$ is the pressure, $Q$ is the uniformly distributed heat generation in ferrofluid layer system, $C_{V, H}$ is the specific of heat capacity at constant volume and magnetic field per unit mass, $\varepsilon$ is the 
porosity, $k$ is the permeability tensor, $k_{1}$ is the thermal conductivity and $\nabla^{2}=\frac{\partial^{2}}{\partial x^{2}}+\frac{\partial^{2}}{\partial y^{2}}+\frac{\partial^{2}}{\partial z^{2}}$ is the Laplacian operator.

Based on Finlayson [35] the Maxwell's equation is given as

$\nabla \cdot \vec{B}=0, \nabla \times \vec{H}=0$ or $\vec{H}=\nabla \varphi$

$\vec{B}=\mu_{0}(\vec{M}+\vec{H})$

where $\vec{B}$ is the magnetic induction, $\vec{H}$ is the magnetic field density, $\vec{M}$ is the magnetization and $\varphi$ is the magnetic potential. Finlayson [35] also state the linearization of magnetic as follows

$\vec{M}=\frac{\vec{H}}{H}\left(M_{0}+\chi\left(H-H_{0}\right)-K\left(T-T_{0}\right)\right)$,

where $\chi=\left(\frac{\partial M}{\partial H}\right)_{H_{0}, T_{0}}$ is the magnetic susceptibility, $K=\left(\frac{\partial M}{\partial H}\right)_{H_{0}, T_{0}}$ is the pyromagnetic co-efficient, $M_{0}=M\left(H_{0}, T_{0}\right), H=|\vec{H}|$ and $M=|\vec{M}|$.

The solutions for the quiescent basic state are as follows

$\vec{q}_{b}=0$

$p_{b}(z)=p_{0}-\rho_{0} h z-\rho_{0} \alpha_{t} h$

$T_{b}(z)=-\beta z+T_{0}$

$\vec{H}_{b}(z)=\left[H_{0}-\frac{K \beta z}{1+\chi}\right] \hat{k}$,

$\vec{M}_{b}(z)=\left[M_{0}+\frac{K \beta z}{1+\chi}\right] \hat{k}$

In order to study the stability of the system, the basic state is perturbed in the following form

$\vec{q}=\vec{q}^{\prime}, p=p_{b}(z)+p^{\prime}, T+T_{b}(z)+T^{\prime}, \vec{H}=\vec{H}_{b}(z)+\vec{H}^{\prime}, \vec{M}=\vec{M}_{b}(z)+\vec{M}^{\prime}$

Substituting Eq. (14) into Eq. (7) and by using equation the basic state, yields

$$
\begin{aligned}
& H_{x}+M_{x}=\left(1+\frac{M_{0}}{H_{0}}\right) H_{x} \\
& H_{y}+M_{y}=\left(1+\frac{M_{0}}{H_{0}}\right) H_{y} \\
& H_{z}+M_{z}=(1+\chi) H_{z}-K T
\end{aligned}
$$

The normal mode expansion is assumed in the form: 
$\{w, T, \varphi\}=\{W(z), \theta(z), \phi(z)\} e^{i(l x+m y)}$,

where $l$ and $m$ are the wave number in $x$ and $y$ direction. Substituted Eq. (14) into momentum equation, energy equation and also Maxwell equation. After that we performing the linearization and eliminate the pressure term by operating the curl twice for the momentum Eq. (4). Followed by the using of Eq. (15) and (16) and non-dimensionalizing the variable by the following setting

$$
\left(x^{*}, y^{*}, z^{*}\right)=\left(\frac{x}{d}, \frac{y}{d}, \frac{z}{d}\right), W^{*}=\frac{d}{v} W, \theta^{*}=\frac{\kappa}{\beta v d} \theta, \phi^{*}=\frac{(1+\chi) \kappa}{K \beta v d^{2}} \phi,
$$

where $v=\mu / \rho_{0}$ is the kinematic viscosity and $\kappa=\kappa_{1} / \rho_{0} c_{0}$ is the thermal diffusivity. After dropping the asterisk, we will get as follows

$$
\begin{aligned}
& {\left[\left(D^{2}-a^{2}\right)^{2}-D a^{-1}\left(\frac{1}{\xi} D^{2}-a^{2}\right)\right] W-a^{2} R m(1+\lambda)(D \phi-\theta)-a^{2} R t(1+\lambda) \theta=0,} \\
& D^{2} \theta-\eta a^{2} \theta-\left(1-M_{2}\right) W=0 \\
& D^{2} \phi-a^{2} M_{3} \phi-D \theta=0,
\end{aligned}
$$

with the boundary condition

$$
\begin{array}{ll}
W=D W=D \theta=\phi=0 & \text { at } z=0 \\
W=D \theta=D \phi=D^{2} W+M a a^{2} \theta=0 & \text { at } z=1,
\end{array}
$$

where

$D=\frac{d}{d z}$

$\lambda$ is the gravity parameter,

$a^{2}$ is the wave number,

$D a=\frac{k_{h}}{d^{2}}$ is the Darcy number,

$\xi=\frac{k_{h}}{k_{v}}$ is an anisotropic permeability,

$R m=R t \cdot M_{1}=\frac{\mu_{0} K_{1}^{2} \beta}{(1+\chi) \alpha_{t} \rho_{0} g}$ is the magnetic Rayleigh number,

$R t=\frac{\alpha_{t} g \beta d^{4}}{v \kappa A}$ is the thermal Rayleigh number,

$\eta=\frac{\kappa_{h}}{\kappa_{v}}$ is an anisotropic effective thermal diffusivity,

$M_{2}=\frac{\mu_{0} T_{0} K_{1}^{2}}{1+\chi}$ is the magnetic parameter,

$M_{3}=\frac{1+\frac{M_{0}}{H_{0}}}{1+\chi}$ is the nonlinearity of the ferrofluid,

$M a=\frac{\sigma_{T} \Delta T d}{\mu \kappa}$ is the Marangoni number

By referring to Finlayson [35], $M_{2}$ will not affect the Benard-Marangoni convection since the value of it will be approximated to zero because the value too small which is $10^{-6}$. To solve Eq. (18) till (20) with the boundary conditions in Eq. (21) and (22), regular perturbation method will be used. The 
variables are in following the form

$$
(W, \theta, \phi)=\left(W_{0}, \theta_{0}, \phi_{0}\right)+a^{2}\left(W_{1}, \theta_{1}, \phi_{1}\right)+\cdots
$$

By substituting Eq. (23) into (18) till (22) we will get the zeroth equation as follows

$$
\begin{aligned}
& D^{4} W_{0}-\left(\frac{1}{D a \xi} D^{2} W_{0}\right)=0 \\
& D^{2} \theta_{0}-W_{0}=0 \\
& D^{2} \phi_{0}-D \theta_{0}=0
\end{aligned}
$$

with the boundary conditions

$$
\begin{array}{ll}
W_{0}=D W_{0}=\theta_{0}=\phi_{0}=0 & \text { at } z=0, \\
W_{0}=D^{2} W_{0}=D \phi_{0}=D \theta_{0}=0 & \text { at } z=1
\end{array}
$$

The solution to the zeroth order Eq. (24) till (26) by using boundary conditions in Eq. (27) and (28) are as follow:

$W_{0}=0, \theta_{0}=1, \phi_{0}=0$.

By substituting Eq. (29) we will get the first order equations as follow

$$
\begin{aligned}
& D^{4} W_{1}-\left(\frac{1}{D a \xi} D^{2} W_{1}\right)+R m(1+\lambda)-R t(1+\lambda)=0 \\
& D^{2} \theta_{1}-\eta-W_{1}=0 \\
& D^{2} \phi_{1}-D \theta_{1}=0
\end{aligned}
$$

with the boundary conditions

$$
\begin{array}{ll}
W_{1}=D W_{1}=D \theta_{1}=\phi_{1}=0 & \text { at } z=0, \\
W_{1}=\phi_{1}=D \theta_{1}=D^{2} W_{1}+M a=0 & \text { at } z=1 .
\end{array}
$$

The Eq. (30) to (34) will be solved by using MAPLE. The equation of $M a_{c}$ will be generate in term of $M_{1}, R t, \eta, \lambda, \xi$ and $D a$. 


\section{Results}

In this document, with the presence of variable gravity, the resulting eigenvalue problem of Marangoni-Benard convection in an anisotropic ferrofluid layer was analytically solved using regular perturbation technique. The boundaries are regarded rigid-free and insulating with a linear stability assessment. The selected values for the gravity parameter suggested by Bala and Chand [32]. The outcomes collected are described graphically in Figure 2-8 to show the effect of different parameters on the critical number of Marangoni, $M a_{c}$ and thermal Rayleigh, $R t_{c}$. From the study, it revealed that $M_{3}$ has a no significant contribution toward the convection of the system and this finding coincides with a previous study from Nanjundappa et al., [36].

Figure 2 demonstrated the impact of various gravity parameter on the onset of BenardMarangoni convection with $R t=1000, M_{1}=1, \eta=1, \xi=0.1$ and $D a=0.001$. The figure clearly shows that the decreasing gravity parameter which is $\lambda=z^{2}-2 z, \lambda=-z$ and $\lambda=-z^{2}$ have stabilizing effect. It contrasts with increasing gravity parameter $\lambda=z$ that promotes the onset of Marangoni convection. The result obtained is in good agreement with the previous study from Bala and Chand [32].

The impact of $M_{1}$ on variable gravity on the onset of Marangoni-Benard convection was noted in Figure 3 The increment of $M_{1}$ will drop the values of $M a_{c}$ and destabilize the system. This situation happens because the increasing of $M_{1}$ will lead to increment of destabilize magnetic force in the system Nanjundappa et al., [36]. The combination of variable gravity parameter $\lambda=-z$ and $M_{1}$ is found to delay the convection while the combination of $\lambda=z$ and $M_{1}$ will enhance the onset of Marangoni-Benard convection.

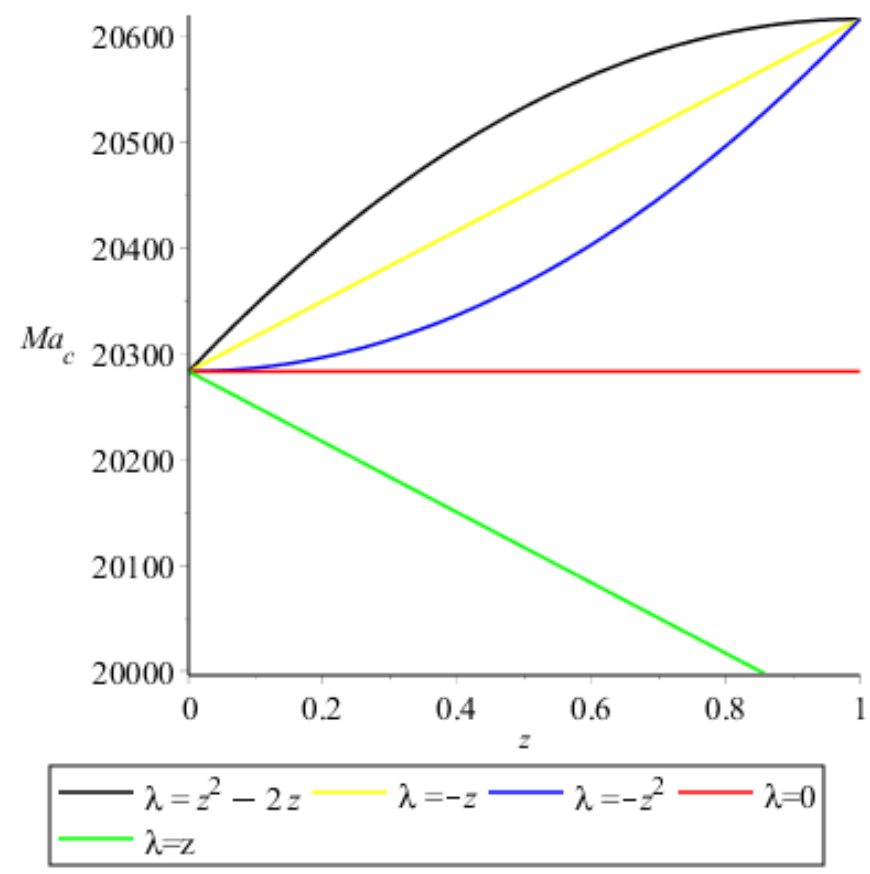

Fig. 2. Stability curve for different value of gravity 


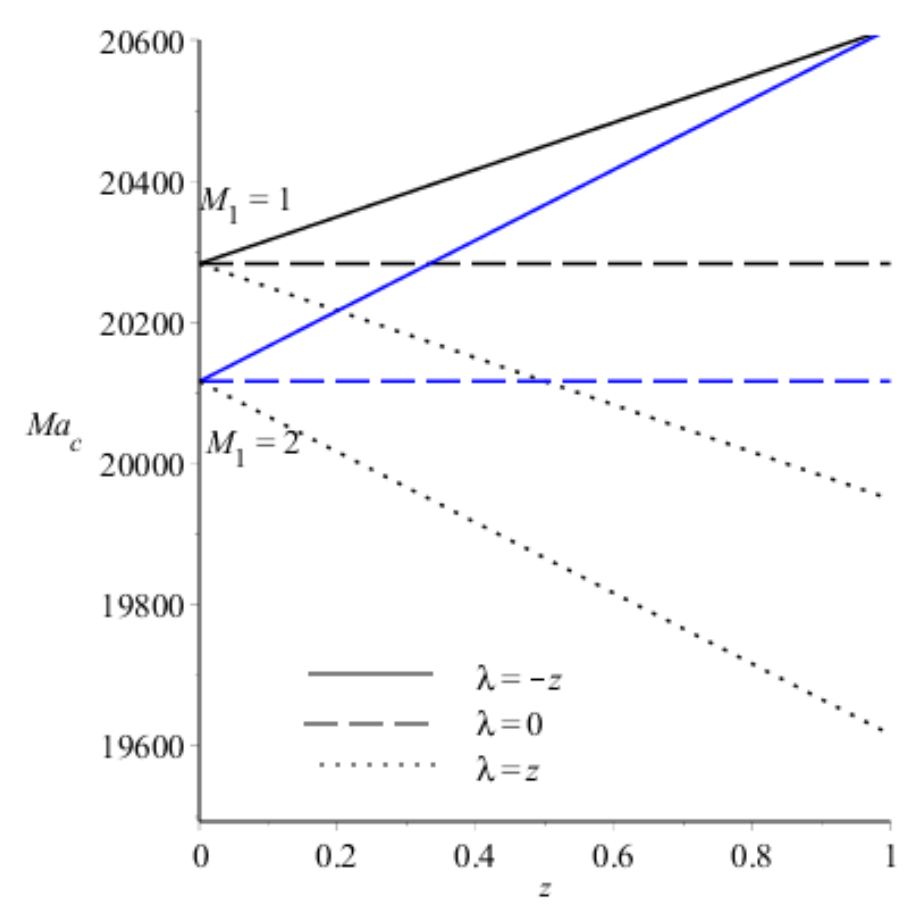

Fig. 3. Stability curve for different value of gravity and $M_{1}$

The impact of two important parameters in anisotropic which is $\xi$ and $\eta$ are depicted in Figure 4 and 5 respectively. For both figures the value of other parameters are $R t=1000, M_{1}=1$ and $D a=$ 0.001. In Figure 4, the $M a_{c}$ values fall as the $\xi$ parameter increases and thus encourages the convection rate in the ferrofluid layer system of an anisotropic porous medium. The reason behind this situation is the increasing of $\xi$ will encouraged the fluid movement in the horizontal direction due to the large horizontal permeability. As an outcome the convection process in an anisotropic medium become unstable (Shivakumara et al., [21]). It is contrast with the effect of $\eta$ in Figure 5, the increasing of $\eta$ lead to elevate the critical Marangoni number and delay the convection.

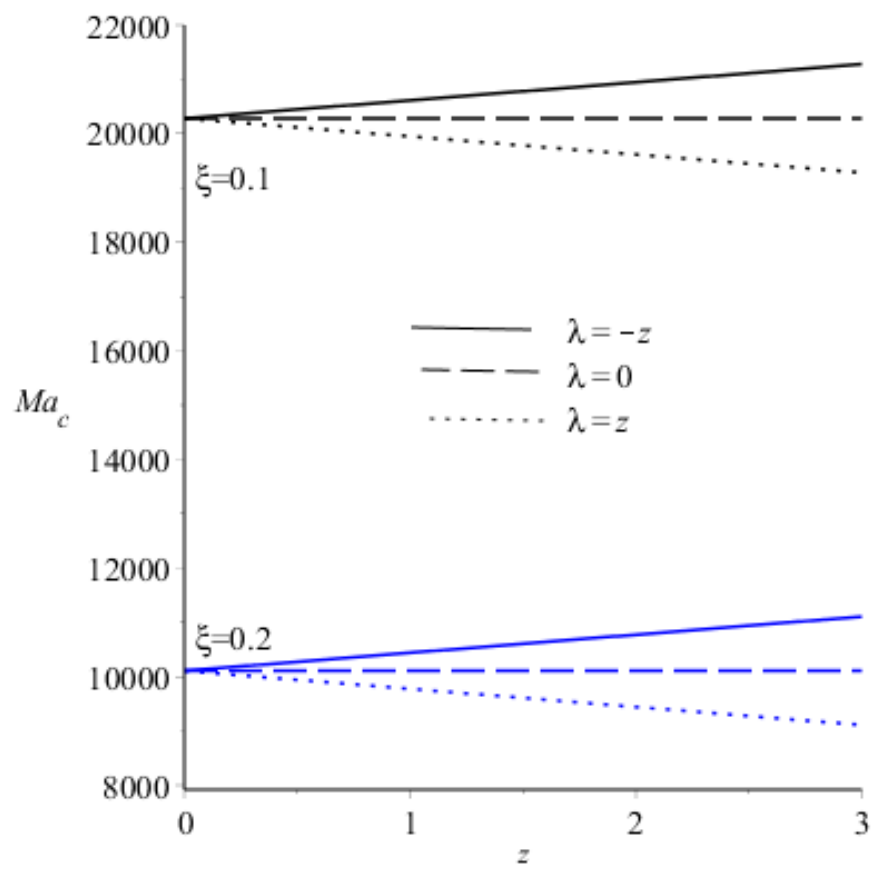

Fig. 4. Stability curve for different value of $\xi$ 


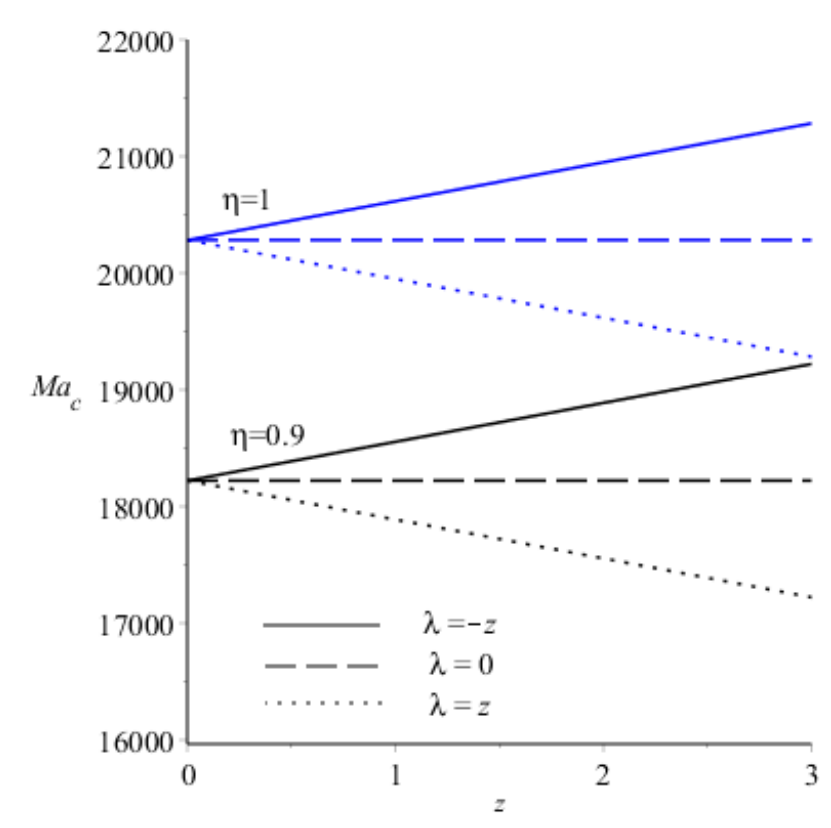

Fig. 5. Stability curve for different value of $\eta$

The combination effects of $M_{1}$ and $\xi$ are illustrated in Figure 6 when $R t=1000, D a=$ $0.001, \lambda=0$ and $\eta=1$. From the graph, it can be seen that boost of both parameters $M_{1}$ and $\xi$ cause a deterioration of the $M a_{c}$ values. This indicates the simultaneous effects of $M_{1}$ and $\xi$ will promotes the convection of Marangoni-Benard in a ferrofluid layer system.

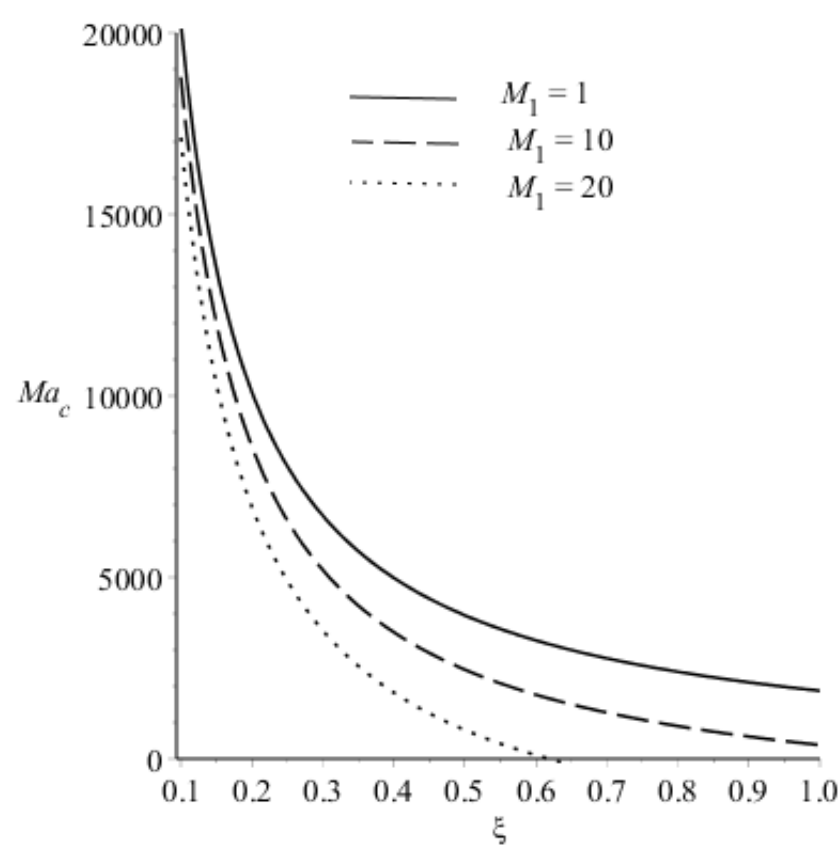

Fig. 6. Impact of $M_{1}$ on $M a_{c}$ against $\xi$

The impact of $M_{1}$ on $D a$ is demonstrate in Figure 7. Other parameters are set $R t=1000, \lambda=$ $0, \eta=1$ and $\xi=0.1$. As reported previously in Figure 3 , the escalation of $M_{1}$ will destabilize the system. This figure also shows the behaviour of $D a$ on $M a_{c}$. It can be seen clearly that the increasing of $D a$ cause the decline of $M a_{c}$ values. It indicates that the increasing of $D a$ will promotes the convection. The same result also reported in Nanjundappa and Vijay Kumar [37]. 


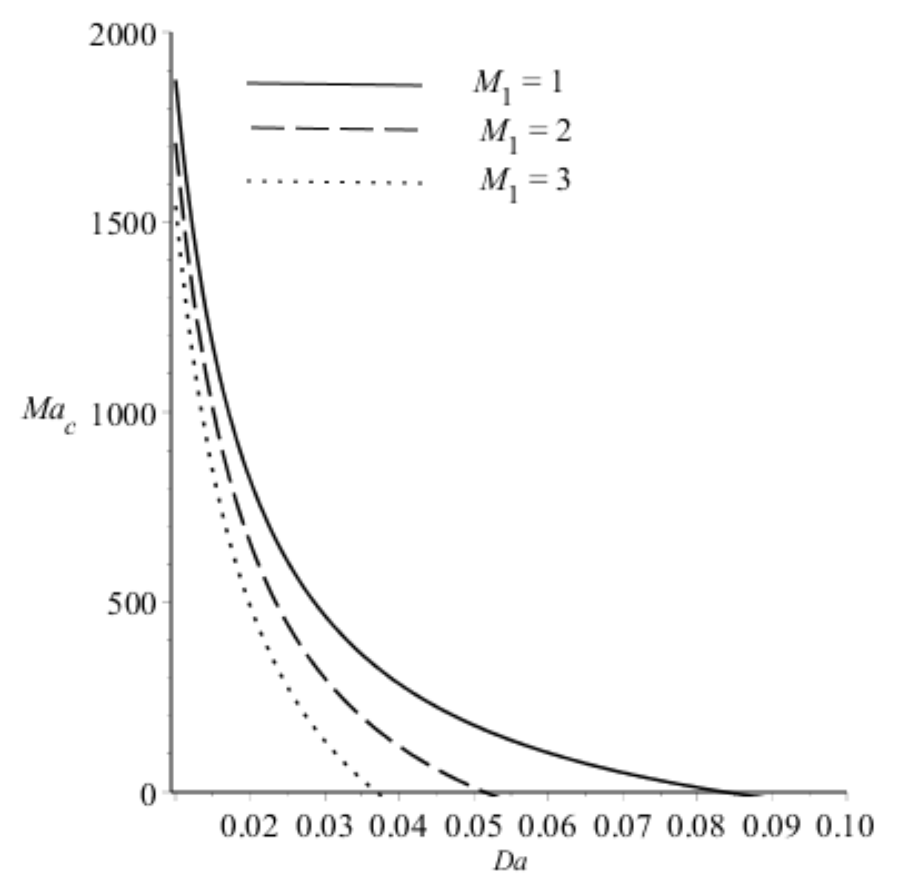

Fig. 7. Impact of $M_{1}$ on $M a_{c}$ against $D a$

Figure 8 shows the response of $M_{1}$ on $R t_{c}$ against $M a_{c}$ for different value of $\lambda$ at $M_{1}=1, \eta=$ $1, \xi=0.1$ and $D a=0.001$. From the graph, it can be seen clearly that the increasing of $M_{1}$ and $M a_{c}$ will lead to compress of $R t_{c} . M_{1}$ value will be merged into a fixed value of $M a$ which is in this study the value is recorded at $M a_{c}=20606.3854$. This situation happens to all $M_{1}$ considered and when $R t=0$ shows that the $R t$ did not affect the convection process Nanjundappa et al., [36].

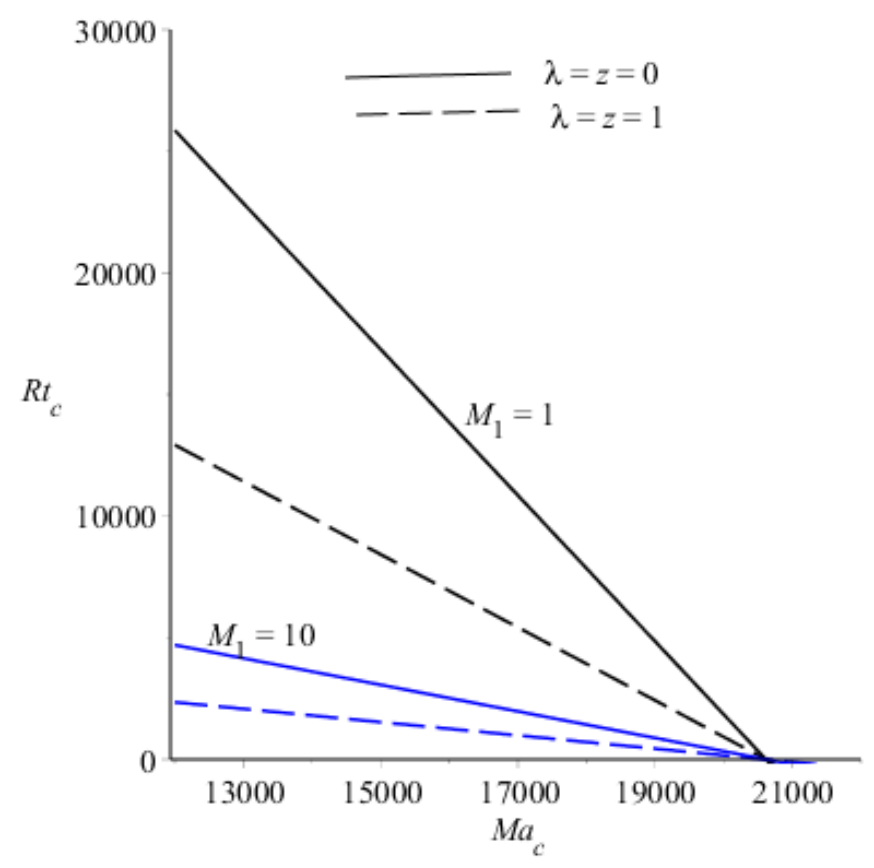

Fig. 8. Impact of $M_{1}$ on $R t_{c}$ against $M a_{c}$ 


\section{Conclusions}

The theoretical investigation into the effects of variable gravity on the onset of MarangoniBenard convection in an anisotropic ferrofluid layer system was conducted. We can conclude that the increasing values of $M_{1}, D a$ and $\xi$ will enhance the convection of a ferrofluid layer system while the increasing of $\eta$ will help to stabilize the system. For the variable gravity, decreasing gravity parameter are found to help in delaying the convection contrast with increasing gravity parameter that promotes the convection.

\section{Acknowledgement}

We would like to thank Universiti Putra Malaysia for all the equipment and financial support. The present research was partially supported by the Putra Grant - Putra Graduate Initiative (IPS)-GPIPS/2018/9642900.

\section{References}

[1] Raju, K. "Effect of temperature dependent viscosity on ferrothermohaline convection saturating an anisotropic porous medium with Soret effect using the Galerkin technique." International journal of heat and technology 36, no. 2 (2018): 439-446.

https://doi.org/10.18280/ijht.360208

[2] Kaiser, Robert, and Gabor Miskolczy. "Some applications of ferrofluid magnetic colloids." IEEE Transactions on magnetics 6, no. 3 (1970): 694-698.

https://doi.org/10.1109/TMAG.1970.1066834

[3] Stiles, Peter J., and Michael Kagan. "Thermoconvective instability of a ferrofluid in a strong magnetic field." Journal of colloid and interface science 134, no. 2 (1990): 435-448. https://doi.org/10.1016/0021-9797(90)90154-G

[4] Hennenberg, Marcel, Boris Weyssow, Slavtcho Slavtchev, V. Alexandrov, and Th Desaive. "Rayleigh-MarangoniBénard instability of a ferrofluid layer in a vertical magnetic field." Journal of magnetism and magnetic materials 289 (2005): 268-271.

https://doi.org/10.1016/i.jmmm.2004.11.076

[5] Mokhtar, Nor Fadzillah Mohd, and Norihan Md Arifin. "Benard-Marangoni ferroconvection with feedback control." In International Journal of Modern Physics: Conference Series, vol. 9, pp. 552-559. World Scientific Publishing Company, 2012.

https://doi.org/10.1142/S201019451200565X

[6] Laroze, David, P. G. Siddheshwar, and Harald Pleiner. "Chaotic convection in a ferrofluid." Communications in Nonlinear Science and Numerical Simulation 18, no. 9 (2013): 2436-2447.

https://doi.org/10.1016/i.cnsns.2013.01.016

[7] Laroze, David, Javier Martinez-Mardones, and Harald Pleiner. "Bénard-Marangoni instability in a viscoelastic ferrofluid." The European Physical Journal Special Topics 219, no. 1 (2013): 71-80. https://doi.org/10.1140/epjst/e2013-01782-6

[8] Prakash, Jyoti, Rajeev Kumar, and Kultaran Kumari. "Thermal convection in a ferromagnetic fluid layer with magnetic field dependent viscosity: A correction applied." Studia Geotechnica et Mechanica 39, no. 3 (2017): 3946.

https://doi.org/10.1515/sgem-2017-0028

[9] Nield, D. A. "Surface tension and buoyancy effects in cellular convection." Journal of Fluid Mechanics 19, no. 3 (1964): 341-352. https://doi.org/10.1017/S0022112064000763

[10] McCaughan, F., and H. Bedir. "Marangoni convection with a deformable surface." (1994): 681-688. https://doi.org/10.1115/1.2901514

[11] Hennenberg, Marcel, M. Ziad Saghir, Alexei Rednikov, and Jean Claude Legros. "Porous media and the BenardMarangoni problem." Transport in porous media 27, no. 3 (1997): 327-355. https://doi.org/10.1023/A:1006564129233

[12] Rudraiah, N., and V. Prasad. "Effect of Brinkman boundary layer on the onset of Marangoni convection in a fluidsaturated porous layer." Acta mechanica 127, no. 1-4 (1998): 235-246. https://doi.org/10.1007/BF01170376 
[13] Saghir, M. Ziad, Paul Comi, and Mehrab Mehrvar. "Effects of interaction between Rayleigh and Marangoni convection in superposed fluid and porous layers." International journal of thermal sciences 41, no. 3 (2002): 207215. https://doi.org/10.1016/S1290-0729(01)01309-6

[14] Shivakumara, I. S., C. E. Nanjundappa, and Krishna B. Chavaraddi. "Darcy-Benard-Marangoni convection in porous media." International journal of heat and mass transfer 52, no. 11-12 (2009): 2815-2823. https://doi.org/10.1016/i.ijheatmasstransfer.2008.09.038

[15] Arifin, Norihan MD, and Nurul Hafizah Zainal Abidin. "Marangoni convection in a variable viscosity fluid layer with feedback control." Journal of Applied Computer Science \& Mathematics 3 (2009): 373-382.

[16] Zhao, Si Cheng, Qiu Sheng Liu, Rong Liu, Henri Nguyen-Thi, and Bernard Billia. "Thermal effects on RayleighMarangoni-Bénard instability in a system of superposed fluid and porous layers." International Journal of Heat and Mass Transfer 53, no. 15-16 (2010): 2951-2954. https://doi.org/10.1016/j.ijheatmasstransfer.2010.04.003

[17] Mokhtar, N. M., N. M. Arifin, R. Nazar, F. Ismail, and M. Suleiman. "Effect of internal heat generation on Marangoni convection in a superposed fluid-porous layer with deformable free surface." International Journal of Physical Sciences 6, no. 23 (2011): 5550-5563.

[18] Mahad, B. A., M. H. B. M. Shariff, M. Isa, and Z. A. Aziz. "Constitutive Model for Anterior Leaflet of Heart with Physical Invariants." Journal of Advanced Research in Applied Mechanics 5, no. 1 (2015): 15-29. http://www.akademiabaru.com/doc/ARAMV5 N1 P15 29.pdf

[19] Degan, G., P. Vasseur, and E. Bilgen. "Convective heat transfer in a vertical anisotropic porous layer." International journal of heat and mass transfer 38, no. 11 (1995): 1975-1987. https://doi.org/10.1016/0017-9310(94)00330-X

[20] Sekar, R., G. Vaidyanathan, and A. Ramanathan. "Ferroconvection in an anisotropic porous medium." International journal of engineering science 34, no. 4 (1996): 399-405. https://doi.org/10.1016/0020-7225(95)00113-1

[21] Shivakumara, I. S., Jinho Lee, and Krishna B. Chavaraddi. "Onset of surface tension driven convection in a fluid layer overlying a layer of an anisotropic porous medium." International journal of heat and mass transfer 54, no. 4 (2011): 994-1001. https://doi.org/10.1016/j.ijheatmasstransfer.2010.10.023

[22] Nanjundappa, C. E., I. S. Shivakumara, Jinho Lee, and M. Ravisha. "The onset of Brinkman ferroconvection in an anisotropic porous medium." International Journal of Engineering Science 49, no. 6 (2011): 497-508. https://doi.org/10.1016/j.ijengsci.2010.12.014

[23] Shivakumara, I. S., S. P. Suma, R. Indira, and Y. H. Gangadharaiah. "Effect of internal heat generation on the onset of Marangoni convection in a fluid layer overlying a layer of an anisotropic porous medium." Transport in porous media 92, no. 3 (2012): 727-743.

https://doi.org/10.1007/s11242-011-9930-7

[24] Bhadauria, B. S. "Double-diffusive convection in a saturated anisotropic porous layer with internal heat source." Transport in porous media 92, no. 2 (2012): 299-320.

https://doi.org/10.1007/s11242-011-9903-x

[25] Capone, Florinda, Maurizio Gentile, and Antony A. Hill. "Convection problems in anisotropic porous media with nonhomogeneous porosity and thermal diffusivity." Acta applicandae mathematicae 122, no. 1 (2012): 85-91. https://doi.org/10.1007/s10440-012-9728-9

[26] Sekar, R., K. Raju, and R. Vasanthakumari. "A linear analytical study of Soret-driven ferrothermohaline convection in an anisotropic porous medium." Journal of Magnetism and Magnetic materials 331 (2013): 122-128. https://doi.org/10.1016/j.jmmm.2012.10.028

[27] Sun, Xiao-hui, Mehrdad Massoudi, Nadine Aubry, Zhi-hua Chen, and Wei-Tao Wu. "Natural convection and anisotropic heat transfer in a ferro-nanofluid under magnetic field." International Journal of Heat and Mass Transfer 133 (2019): 581-595. https://doi.org/10.1016/j.ijheatmasstransfer.2018.12.132

[28] Hamid, Nur Zarifah Abdul, Nor Fadzillah Mohd Mokhtar, Norihan Md Arifin, Mohammad Hasan, and Abdul Sathar. "Effect of Nonlinear Temperature Profile on Thermal Convection in a Binary Fluid Saturated an Anisotropic Porous Medium." studies 56, no. 1 (2019): 43-58.

[29] Rionero, S., and B. Straughan. "Convection in a porous medium with internal heat source and variable gravity effects." International Journal of Engineering Science 28, no. 6 (1990): 497-503.

https://doi.org/10.1016/0020-7225(90)90052-K 
[30] Alex, Sherin M., Prabhamani R. Patil, and K. S. Venkatakrishnan. "Variable gravity effects on thermal instability in a porous medium with internal heat source and inclined temperature gradient." Fluid Dynamics Research 29, no. 1 (2001): 1.

https://doi.org/10.1016/S0169-5983(01)00016-8

[31] Chand, Ramesh, and Arvind Kumar. "Thermal instability of rotating Maxwell visco-elastic fluid with variable gravity in porous medium." International Journal of Advances in Applied Mathematics and Mechanics 1, no. 2 (2013): 3038.

[32] Bala, Ankuj, and Ramesh Chand. "Variable gravity effect on the thermal instability of Ferrofluid in a Brinkman porous medium." International Journal of Astronomy, Astrophysics and Space Science 2, no. 5 (2015): 39.

[33] Chand, Ramesh, G. C. Rana, and S. K. Kango. "Effect of variable gravity on thermal instability of rotating nanofluid in porous medium." FME Transactions 43, no. 1 (2015): 62-69.

https://doi.org/10.5937/fmet1501062c

[34] Varshney, A.K. "Thermal Stability Of A Fluid Layer In A Variable Gravitational Field In Porous." Journal of Advanced Computing and Communication Technologies 5, no. 3 (2017): 55-60.

[35] Finlayson, B. A. "Convective instability of ferromagnetic fluids." Journal of Fluid Mechanics 40, no. 4 (1970): $753-$ 767.

https://doi.org/10.1017/S0022112070000423

[36] Nanjundappa, C. E., I. S. Shivakumara, and R. Arunkumar. "Onset of Bénard-Marangoni ferroconvection with internal heat generation." Microgravity Science and Technology 23, no. 1 (2011): 29-39. https://doi.org/10.1007/s12217-010-9218-5

[37] Nanjundappa, C. E., and B. Vijay Kumar. "PENETRATIVE FERROCONVECTION VIA INTERNAL HEATING IN A FERROFLUID ANISOTROPIC POROUS MEDIUM." Magnetohydrodynamisc 49,no.3 (2013): 441-47. https://doi.org/10.22364/mhd.49.3-4.36 